\title{
Probiotic (Enterococcus faecium) induced responses of the hepatic proteome improves metabolic efficiency of broiler chickens (Gallus gallus)
}

Aijuan Zheng ${ }^{1+}$, Jianjie Luo ${ }^{1+}$, Kun Meng ${ }^{1}$, Jianke Li ${ }^{2}$, Wayne L. Bryden ${ }^{3}$, Wenhuan Chang ${ }^{1}$, Shu Zhang ${ }^{1}$, L. X. N. Wang ${ }^{3}$, Guohua Liu ${ }^{1 *}$ and Bin Yao ${ }^{1 *}$

\begin{abstract}
Background: The liver plays important roles in nutrient metabolism, detoxification and immunity. Enterococcus faecium (E. faecium) is a probiotic that has been shown to have positive effects on broiler production. However, its molecular effects on liver metabolism have not been characterized. This study aims to further identify the biological roles of $E$. faecium by characterizing the hepatic proteomic changes of broilers (Gallus gallus) fed E. faecium using two-dimensional fluorescence difference gel electrophoresis (2-D DIGE) and mass spectrometry (MS).

Results: Thirty-three proteins (50 protein spots) involved in nutrient metabolism, immunity and the antioxidant system were shown to be differentially expressed in the liver of broilers fed E. faecium than from birds not fed the probiotic. The biological processes of sulphur amino acids, vitamin and cellular hormone metabolism, sulphur compound biosynthesis and protein tetramerization were enhanced in the liver of broilers fed E. faecium. However, proteins involved in calcium ion flux, cell redox homeostasis and platelet activation related to hepatic immune responses were down-regulated in broilers fed E. faecium. These results indicate that the supplementation of poultry feed with E. faecium may alter the partitioning of nutrients and promote optimal nutrient utilization.

Conclusions: This study assists in unraveling the molecular effects of the dietary probiotic, E. faecium, in the liver of broiler chickens. It shows that the probiotic improves the metabolism of nutrients and decreases inflammatory responses. Our findings extend previous knowledge of the mechanism of dietary probiotic action and provide new findings for research and future probiotic development.
\end{abstract}

Keywords: Broiler chicken (Gallus gallus), Enterococcus faecium, Liver, Proteome, Probiotics

\section{Background}

Probiotics are live, non-pathogenic microorganisms that can regulate immune responses and suppress inflammation of intestinal cells [1]. In so doing, probiotics have positive effects on the gut microbiota [2]. Probiotics, along with plant extracts [3] and organic acids [4] are being increasingly used in chicken (broiler) meat production as alternatives to antibiotic growth promoters to

\footnotetext{
*Correspondence: liuguohua@caas.cn; binyao@caas.cn

${ }^{\dagger}$ Equal contributors

${ }^{1}$ Key Laboratory of Feed Biotechnology of Ministry of Agriculture, Feed

Research Institute, Chinese Academy of Agricultural Sciences, Beijing 100081,

People's Republic of China

Full list of author information is available at the end of the article
}

improve broiler performance and enhance the sustainability of production. The application of antibiotics as growth promoters has been prohibited in the European Union because of increased antibiotic resistance [5] and in response to consumers demands for safe, and quality broiler meat products.

Enterococcus faecium is a facultative anaerobic lactic acid bacterium found in the intestinal microbiota of humans and animals [6]. As one of the direct-fed microorganism strains recognized by the Association of American Feed Control Officials, E. faecium is permitted as a probiotic supplement for broiler chicken diets [7, 8]. Its impact on broiler chickens include promotion of 
immune organ development [9], increased diversity of the gut microbial population [10], resistance to infection [11], changes of antioxidant status [7, 10], and increased intestinal absorptive surface area [10, 12, 13]. The latter two effects have also been demonstrated in our previous studies of the proteome responses of the intestinal mucosa of broilers fed E. faecium [9]. We have also shown that this probiotic induced proteome changes that improved broiler meat quality and yield [14].

The liver plays a central role in nutrient metabolism, including glycogen storage, protein and lipid synthesis, detoxification, and production of chemicals necessary for digestion $[15,16]$. There are many reports that the intestinal microbiota and its products indirectly affect the liver $[6,7,9-11,13]$. As a 'first pass' organ exposed to the highest concentration of nutrients and other substances in the portal system, the liver is most vulnerable to their effects and represents a target organ to assess the effects of probiotics. Undoubtedly, studying variations of the hepatic proteome following dietary probiotic supplementation will provide further insights into the mechanisms of probiotic action. Thus, the aim of this study was to undertake a molecular characterization of hepatic metabolism of broiler chickens fed the probiotic E. faecium

\section{Methods}

The study described in this paper was conducted in the Feed Research Institute, Chinese Academy of Agricultural Sciences (CAAS), Beijing, China. The care and use of all birds in this experiment was approved by the Animal Care and Use Committee of the Feed Research Institute of CAAS.

\section{Materials and chemical reagents}

Microcapsules of E. faecium CGMCC 2516 [17, 18] (viable count $\geq 1 \times 10^{10} \mathrm{cfu} / \mathrm{g}$; Challenge Biotechnology Ltd. Co., Beijing, China) were used in the present experiment. All reagents for two-dimensional fluorescence difference gel electrophoresis (2-D DIGE) were purchased from BioRad (Hercules, CA), Roche (Mannheim, Germany), GE Healthcare (Uppsala, Sweden) and Sigma-Aldrich (St. Louis, MO). The reagents for LC-Chip-ESI-QTOF-MS were purchased from Bruker Daltonics (Billerica, MA), Roche and J. T. Baker (Phillipsburg, NJ). 2-D Quant Kit was purchased from GE Healthcare.

\section{Bird management and dietary treatments}

Arbor Acres (AA) broilers were purchased from the Huadu Chicken Co. (Beijing, China). A total of 216, 1day-old, male AA broiler chicks were randomly divided into two groups, control and treatment. Each group had 9 replicates (cage) and each replicate contained 12 birds. The distribution of cages was arranged to avoid any location effects on the poultry house. The chickens were reared in two stages, starter (0-21 days) and grower (22-42 days), and fed appropriate corn-soybean meal diets (Additional file 1: Table S1) containing $1.0 \times 10^{6} \mathrm{cfu} /$ $\mathrm{g}$ (starter diet) and $1.2 \times 10^{6} \mathrm{cfu} / \mathrm{g}$ (grower diet) of $E$. faecium (treatment) or not (control) for 42 days. In accordance with the AA Broiler Management Guide (Aviagen Group 2009), all chicks were inoculated and subjected to a photoperiod of $23 \mathrm{~h} \mathrm{light}$ on days $0-7$ and $20 \mathrm{~h}$ thereafter. The room temperature was $33-35{ }^{\circ} \mathrm{C}$ for days $0-3$ and gradually reduced to $20{ }^{\circ} \mathrm{C}$ by day 28 . Relative humidity was maintained at $60-70 \%$ during the first week and then at $50-60 \%$ for the remainder of the study.

\section{Sample preparation}

On day 42, chickens from each group were randomly selected, electrically stunned, and manually slaughtered within 5 min [19]. The livers were removed and washed with PBS $\left(\mathrm{NaCl} 8 \mathrm{~g} / \mathrm{L}, \mathrm{Na}_{2} \mathrm{HPO}_{4} 1.44 \mathrm{~g} / \mathrm{L}, \mathrm{KH}_{2} \mathrm{PO}_{4}\right.$ $240 \mathrm{mg} / \mathrm{L}$, and $\mathrm{KCl} 200 \mathrm{mg} / \mathrm{L}, \mathrm{pH} 7.2$ ) to remove any blood and contaminants on the surface and immediately stored in liquid nitrogen [20]. Hepatic protein extraction was performed as described previously with some modifications [14]. Liver samples (100 mg) were homogenized with liquid nitrogen and dissolved in $1 \mathrm{~mL}$ of PBS (pH 7.0) containing EDTA-free protease inhibitor cocktail tablets (Roche). The proteins insoluble in PBS were extracted by lysis buffer ( $9 \mathrm{M}$ urea, $2 \mathrm{M}$ thiourea, $4 \%$ CHAPS, 2 EDTA-free protease inhibitor cocktail tablets, $\mathrm{pH}$ 8.5) and combined with the PBS soluble proteins. Trichloroacetic acid was added at a ratio of 1:9, followed by 10 -min incubation at $-20{ }^{\circ} \mathrm{C}$. After centrifugation at $15,000 \times g$ at $4{ }^{\circ} \mathrm{C}$ for $10 \mathrm{~min}$, the pellet was washed with cold acetone, incubated and re-centrifuged as described above. The pellet was then air dried, suspended in lysis buffer at the ratio of $1 \mathrm{mg}: 10 \mu \mathrm{L}$. The protein concentration of the supernatant was determined by the 2-D Quant Kit.

\section{2-D DIGE and image analysis}

The $\mathrm{pH}$ of the proteins was adjusted to 8.5 with $50 \mathrm{mM}$ $\mathrm{NaOH}$, and the concentration was adjusted to $5 \mathrm{mg} / \mathrm{mL}$ with lysis buffer. Equal amounts of proteins from the 6 samples of each control and treatment group were pooled together as the internal standard. The proteins $(50 \mu \mathrm{g})$ were then labeled individually with $400 \mathrm{pmol}$ of Cy3, Cy 5 or Cy2 (specific for internal standard) on ice for $30 \mathrm{~min}$ in the dark and then quenched with $1 \mu \mathrm{L}$ of $10 \mathrm{mM}$ lysine on ice for another $10 \mathrm{~min}$. 2-D DIGE was performed as described with some modifications [21]. To avoid erroneous conclusions due to individual variations, the same quantity of proteins from the liver of three chickens were pooled as a biological replicate, and three biological replicates were acquired for each group. 
Three 2-D DIGE gels were independently carried out with the same internal standard sample. The Cy3- and Cy5-labeled proteins $(50 \mu \mathrm{g})$ were combined, and then mixed with $50 \mu \mathrm{g}$ of Cy2-labeled internal standard. An equal volume of $2 \times$ sample buffer $(9 \mathrm{M}$ urea, $2 \mathrm{M}$ thiourea, $4 \%$ CHAPS, $130 \mathrm{mM}$ DTT, and $1 \%$ IPG buffer, $\mathrm{pH}$ 3.0-10.0; GE Healthcare) was then added to the sample, followed by the addition of rehydration buffer (8 M urea, 2 \% CHAPS, 45 mM DTT, 0.5 \% IPG buffer, and a trace amount of bromophenol blue, $\mathrm{pH} 3.0-10.0$ ) to a total volume of $450 \mu \mathrm{L}$. Samples were applied to 24$\mathrm{cm} \mathrm{pH} \mathrm{3.0-10.0} \mathrm{IPG} \mathrm{strips} \mathrm{(Bio-Rad),} \mathrm{and} \mathrm{isoelectric} \mathrm{fo-}$ cusing was performed using the IPGphor IEF system (GE Healthcare). The isoelectric focusing program was set as follows: $50 \mathrm{~V}$ for $14 \mathrm{~h}$, Grd $500 \mathrm{~V}$ for $30 \mathrm{~min}$, Step $500 \mathrm{~V}$ for $1 \mathrm{~h}$, Grd $1000 \mathrm{~V}$ for $30 \mathrm{~min}$, Step $1000 \mathrm{~V}$ for $1 \mathrm{~h}$, Grd $8000 \mathrm{~V}$ for $3 \mathrm{~h}$, and step $8000 \mathrm{~V}$ for $30000 \mathrm{Vh}$. The IPG strips on the concentrator were equilibrated in buffer A (375 mM Tris- $\mathrm{HCl}$ [pH 8.8], $6 \mathrm{M}$ urea, $29.3 \%$ glycerol, 2 \% SDS, 1 \% DTT and a trace amount of bromophenol blue) for $15 \mathrm{~min}$ at room temperature and followed by equilibration with buffer B $(375 \mathrm{mM}$ Tris$\mathrm{HCl}$ [pH 8.8], $6 \mathrm{M}$ urea, 29.3 \% glycerol, 2 \% SDS, $2.5 \%$ iodoacetamid and a trace amount of bromophenol blue) for another 15-min incubation at room temperature.

Homogeneous polyacrylamide gels (12\%) were precast with low fluorescence glass plates using an Ettan DALT six-gel caster, and IPG strips were placed on top of it. Strips were overlaid with $0.5 \%$ Agarose-LE (Affymatrix, Santa Clara, CA) in $1 \times$ running buffer containing bromphenol blue and were run for 14-16 h (2 W per gel, overnight) at $16{ }^{\circ} \mathrm{C}$ in an Ettan DALT six electrophoresis system (GE Healthcare). All electrophoresis procedures were performed in dim light or in the dark. After the run was completed, the 2-D DIGE gels were scanned in situ using a Typhoon 9410 Variable Mode Imager (GE Healthcare) and analyzed by the DeCyder Differential Analysis Software (version 7.0, GE Healthcare) according to the manufacturer's instructions.

\section{Identification of protein spots of differential abundance by LC-Chip ESI-QTOF-MS}

The identification of protein spots was carried out as described by Begna et al. with some modifications [22]. Interesting protein spots from the preparative gels were in-gel digested and identified by LC-Chip ESI-QTOFMS (Q-TOF 6520, Agilent, Santa Clara, CA). The tandem mass spectra were retrieved using the Mass Hunter software (Version B.02.01, Agilent). Before the MS/MS data search, a peak-list was generated by the Mascot Distiller software (Version 3.2.1.0, Matrix Science, Boston, MA). The MS/MS data were searched against Mascot 2.2 (Matrix Science) applied to NCBInr (released March 2015) with the following parameters: carbamidomethylation (C) and oxidation $(\mathrm{M})$ were the fixed and variable modifications, respectively; taxonomy, all entries; enzyme, trypsin/ $\mathrm{P}$; missed cleavages, 1 ; peptide tolerance, $\pm 20 \mathrm{ppm}$; and MS/MS tolerance, $\pm 0.02 \mathrm{Da}$. When the identified peptides were matched to multiple members of a protein family, or a protein appeared under the same name and accession number, the match was made in terms of the higher Mascot score, the putative function, and the differential patterns of the protein spots on the 2-D DIGE gels. Protein identifications were accepted if they established a probability greater than $95 \%$ and contained at least two identified peptides having maximal peptide coverage. The relative abundances of differentially expressed proteins were normalized by the internal standard of pooled Cy2-labeled proteins.

Bioinformatics analysis of differentially abundant proteins The ClueGo software http://apps.cytoscape.org/apps/ cluego with the Gene Ontology (GO) database (released March 2015) and Kyoto encyclopedia of genes and genomes (KEGG) database (released March, 2015) was used to classify identified proteins into specific functional terms and a pathway enrichment analysis. The gene ontology analysis based on biological process and enrichment analysis was performed by the right-side hyper-geometric statistic test and its probability value was corrected by the Bonferroni's method [23]. While pathway enrichment analysis was performed by using the ClueGo software and the Gallus gallus database from KEGG database.

A protein interaction network of differential proteins was analyzed using the online database resource Search Tool (http://string-db.org/) for the Retrieval of Interacting Genes (STRING 9.05) [24]. The protein regulation networks and protein interaction maps are in the Gallus gallus molecular networks database. The network nodes are the proteins, and the edges represent the predicted functional associations. An edge may be drawn with up to seven differently colored lines-these lines representing the existence of seven types of evidence used in interaction prediction. The interactions between the imported proteins and all proteins stored in the database were then identified.

\section{Validation of proteins of differential abundance by qPCR}

To further understand the relationship between proteins and their encoding genes, qPCR was run for proteins of differential hepatic abundance at the mRNA level. Specific primers for target genes of the identified proteins were designed using the primer BLAST of NCBI and nucleotide information in GenBank (Additional file 2: Table S2). Total RNA was prepared from the liver of control and treated groups using TRNzol-A+ (TIANGEN, Beijing, China). RNA quality and concentration were detected using spectrophotometer (Ultrospec 2100 
pro, GE Healthcare) and agarose gel electrophoresis. cDNA synthesis with $5 \mu \mathrm{g}$ of RNA was performed using the Fast Quant RT Kit (with gDNase) (TIANGEN). qPCR was conducted using the iCycler iQ5 system. The PCR was performed in a $20-\mu \mathrm{L}$ reaction system containing $1 \mu \mathrm{L}$ of cDNA, $0.5 \mu \mathrm{L}$ of each primer $(10 \mu \mathrm{M}), 10 \mu \mathrm{L}$ of Super Real PreMix (SYBR Green) (TIANGEN) and $8.2 \mu \mathrm{L}$ of water. The fold-change was calculated using the IQTM5 software (Bio-Rad) with the $2^{-\Delta \Delta \mathrm{Ct}}$ method [25]. All operation for qPCR was followed by the MIQE [26].

\section{Results}

The broiler chickens were clinically normal throughout the experiment. As reported previously, dietary supplementation with $E$. faecium did not significantly increase growth rate or feed intake of the broilers. However, feed conversion efficiency was improved [9], along with meat quality and yield [14].

\section{Identification of proteins of differential abundance}

A total of 213 protein spots were detected on 2-D DIGE gels of liver, with molecular weights and $p \mathrm{I}$ ranging from 10 to $100 \mathrm{kDa}$ and 3.0 to 10.0 , respectively (Fig. 1). There were 58 protein spots that displayed significantly different expression (1.4-fold, $p<0.05)$ between control and treatment groups. Fifty protein spots $(86 \%)$ were identified by MS (Table 1), with remainder unidentified, due to weak spectra.

\section{GO and KEGG function enrichment analysis}

The GO annotation was used to determine the biological events behind the data and to provide a primary overview of the hepatic proteome. Use of the ClueGo software allowed the functional enrichment analysis based on biological processes (Fig. 2). Five major functional groups were significantly enriched, i.e., amino acid metabolism, lipid metabolism, vitamin metabolism, nucleotide metabolism, and immunity and antioxidant system. With the sulphur amino acid biosynthetic process as the leading term (a term highly statistically significant or with the lowest $p$-value), proteins involved in amino acid metabolism contained BHMT (betaine-homocysteine Smethyltransferase 1 , spots 24 and 41), CTH (cystathionase, spot 33), and GOT1 (aspartate aminotransferase, spot 32). Similarly, with cell redox homeostasis as the leading term, the immunity and antioxidant system functional group contained CAT (catalase, spots 12 and 13), FGB (fibrinogen $\beta$ chain, spot 14) and FGG (fibrinogen gamma chain, spots 16 and 18). With the cholesterol metabolic process as the leading term, the lipid metabolism functional group contained AKR1D1 (3-oxo-5- $\beta$-steroid 4-dehydrogenase isoform 2, spot 35) and APOA1 (apolipoprotein A-I, spot 48). With the vitamin metabolic process as the leading term, the vitamin metabolism

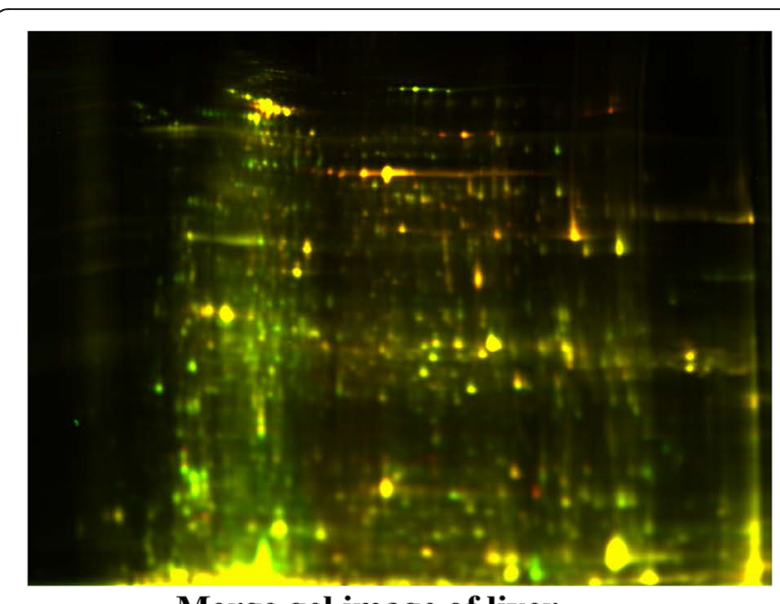

Merge gel image of liver



Liver of treatment group

Fig. 1 2D-DIGE hepatic protein profiles of broilers fed the probiotic $E$. faecium or not. Protein spots showing significant differences (1.4-fold, $p<0.05)$ were cut out and identified by LC-Chip-ESI-QTOF-MS. Proteins of differential abundance with known identities are number-labeled and marked red and green for up-regulation and

down-regulation, respectively

functional group contained ALDH1A1 (Retinal dehydrogenase 1, spot 28) and RGN (regucalcin, spot 38). The nucleotide metabolism functional group contained ATIC (bifunctional purine biosynthesis protein PURH, spot 8) and DPYS (dihydropyrimidinase, spot 53). The supplementation with E. faecium significantly up-regulated the sulphur amino acid biosynthetic and metabolic process, vitamin and cellular hormone metabolic process and protein tetramerization in the liver of broiler chickens. However, the biological process of glycerol ether and organic ether metabolism, cell redox homeostasis, platelet activation and response to calcium ion were down-regulated by dietary supplementation with $E$. faecium.

The KEGG pathway analysis showed that eight differentially expressed proteins were significantly enriched in the five pathways, which participate in multiple biological processes (Table 2). These pathways were mainly 
Table 1 Differentially expressed proteins detected in the liver of AA broiler chickens fed the dietary probiotic E. facecium ${ }^{\text {a }}$

\begin{tabular}{|c|c|c|c|c|c|c|c|c|c|}
\hline Spot no. & Protein name & Accession no. & Symbol ID & $\operatorname{Mr}(\mathrm{kDa}) / \mathrm{pl}$ & Sequence coverage (\%) & Matched/searched & Mascot score & Av. ratio (treatment/control) & $p$ value \\
\hline \multicolumn{10}{|c|}{ Immune and antioxidant system } \\
\hline 1 & Transferrin & gi|83754919 & LTF & $77.49 / 6.70$ & 50 & $75 / 326$ & 1282 & -1.59 & $7.6 \mathrm{E}-03$ \\
\hline 2 & Transferrin & gi|83754919 & LTF & $77.49 / 6.70$ & 50 & $75 / 326$ & 1282 & -1.60 & $1.4 \mathrm{E}-02$ \\
\hline 3 & PIT 54 protein & gi|46395491 & PIT54 & $52.67 / 4.61$ & 23 & $16 / 252$ & 272 & -1.61 & $8.5 E-04$ \\
\hline 4 & Serum albumin precursor & gi|45383974 & ALB & $71.87 / 5.51$ & 76 & $96 / 411$ & 2211 & 2.10 & $1.2 \mathrm{E}-02$ \\
\hline 5 & Serum albumin precursor & gi|45383974 & ALB & $71.87 / 5.51$ & 80 & $118 / 411$ & 2390 & 1.73 & $2.0 E-02$ \\
\hline 6 & Serum albumin precursor & gi|45383974 & ALB & $71.87 / 5.51$ & 80 & $100 / 411$ & 2671 & 2.39 & $3.4 \mathrm{E}-03$ \\
\hline 7 & PIT54 protein & gi|46395491 & PIT 54 & $52.67 / 4.61$ & 23 & $16 / 252$ & 272 & -1.67 & $6.7 E-05$ \\
\hline 14 & Fibrinogen $\beta$ chain & gi|399491 & FGB & $53.27 / 7.18$ & 46 & $29 / 222$ & 619 & -1.56 & $3.2 E-03$ \\
\hline 15 & Serum albumin precursor & gi|45383974 & ALB & $71.87 / 5.51$ & 31 & $23 / 263$ & 379 & 1.47 & $1.8 \mathrm{E}-02$ \\
\hline 16 & Fibrinogen gamma chain & gil8569623 & FGG & $47.49 / 5.40$ & 28 & $19 / 246$ & 296 & -1.57 & $1.5 E-02$ \\
\hline 18 & Fibrinogen gamma chain & gi|8569623 & FGG & $47.49 / 5.40$ & 26 & $12 / 191$ & 232 & -1.70 & $5.8 \mathrm{E}-04$ \\
\hline 12 & Catalase & gi|53127216 & CAT & $60.28 / 8.09$ & 39 & $39 / 290$ & 685 & 2.14 & $8.0 E-03$ \\
\hline 13 & Catalase & gi|53127216 & CAT & $60.28 / 8.09$ & 67 & $68 / 334$ & 1104 & 2.39 & $1.4 \mathrm{E}-03$ \\
\hline 34 & Epoxide hydrolase 2 & gi|75832164 & $\mathrm{EPHX} 2$ & $63.72 / 5.89$ & 19 & $18 / 201$ & 308 & 1.43 & $4.2 \mathrm{E}-02$ \\
\hline 40 & HSP108 & gil63509 & HSP90B1 & $91.45 / 4.81$ & 4 & $8 / 241$ & 86 & -1.69 & $1.1 E-02$ \\
\hline 46 & HSP108 & gi|63509 & HSP90B1 & $91.45 / 4.81$ & 3 & $2 / 241$ & 56 & -1.48 & $1.8 \mathrm{E}-02$ \\
\hline 49 & Annexin A6 & gi|50982399 & ANXA6 & $75.58 / 5.57$ & 8 & $4 / 244$ & 71 & -1.64 & $2.3 \mathrm{E}-03$ \\
\hline 50 & GlutathioneS-transferase 2 & gi|2981970 & GSTM2 & 25.92/7.00 & 63 & $18 / 217$ & 546 & 1.48 & $3.9 E-03$ \\
\hline 51 & HSP108 & gi|63509 & HSP90B1 & $91.45 / 4.81$ & 13 & $16 / 209$ & 402 & -1.76 & $2.4 \mathrm{E}-02$ \\
\hline \multicolumn{10}{|c|}{$\begin{array}{l}\text { Carbohydrate metabolism and energy } \\
\text { production }\end{array}$} \\
\hline 20 & a-Enolase & gi|46048768 & ENO1 & $47.62 / 6.17$ & 48 & $43 / 296$ & 882 & 1.94 & $3.2 E-03$ \\
\hline 21 & a-Enolase & gi|46048768 & ENO1 & $47.62 / 6.17$ & 38 & $17 / 227$ & 378 & 1.78 & $4.8 E-03$ \\
\hline 23 & a-Enolase & gi|46048768 & ENO1 & $47.62 / 6.17$ & 52 & $28 / 279$ & 821 & 1.76 & $5.2 E-03$ \\
\hline 25 & a-Enolase & gi|46048768 & ENO1 & $47.62 / 6.17$ & 41 & $39 / 291$ & 880 & 1.77 & $1.3 \mathrm{E}-02$ \\
\hline 26 & a-Enolase & gi|46048768 & ENO1 & $47.62 / 6.17$ & 53 & $52 / 324$ & 1088 & 1.80 & $6.6 \mathrm{E}-03$ \\
\hline 27 & $\begin{array}{l}\text { Mitochondrial inner membrane } \\
\text { protein }\end{array}$ & gi|57530041 & IMMT & $79.54 / 5.72$ & 6 & $4 / 266$ & 50 & -1.74 & $5.4 \mathrm{E}-03$ \\
\hline 36 & a-Enolase & gi|46048768 & ENO1 & $47.62 / 6.17$ & 11 & $5 / 290$ & 93 & 1.44 & $4.3 \mathrm{E}-04$ \\
\hline 37 & Phosphoglycolate phosphatase & gi|71894743 & PGP & $33.55 / 5.53$ & 33 & $15 / 271$ & 364 & 1.50 & $9.4 \mathrm{E}-03$ \\
\hline 44 & a-Enolase & gi|46048768 & ENO1 & $47.62 / 6.17$ & 8 & $4 / 296$ & 62 & -1.41 & $3.9 E-02$ \\
\hline 47 & a-Enolase & gi|46048768 & ENO1 & $47.62 / 6.17$ & 23 & $11 / 240$ & 313 & -1.65 & $2.1 \mathrm{E}-02$ \\
\hline
\end{tabular}


Table 1 Differentially expressed proteins detected in the liver of AA broiler chickens fed the dietary probiotic E. facecium ${ }^{\text {a }}$ (Continued)

\begin{tabular}{|c|c|c|c|c|c|c|c|c|c|}
\hline 55 & Alcohol dehydrogenase 6 & gi|45384164 & $\mathrm{ADH} 6$ & $40.89 / 7.85$ & 16 & $8 / 231$ & 130 & 3.38 & $2.2 E-03$ \\
\hline 56 & $\begin{array}{l}\text { Phosphoenolpyruvate } \\
\text { carboxykinase [GTP], } \\
\text { mitochondrial }\end{array}$ & gi|45382653 & PCK2 & $71.72 / 8.16$ & 11 & $9 / 196$ & 134 & 1.65 & $7.8 \mathrm{E}-03$ \\
\hline 58 & Phosphoglycerate kinase & gi|45384486 & PGK1 & $45.09 / 8.31$ & 15 & $14 / 256$ & 333 & -1.45 & $1.5 \mathrm{E}-02$ \\
\hline \multicolumn{10}{|c|}{ Amino acid and protein metabolism } \\
\hline 17 & $\begin{array}{l}\text { Alanyl-tRNA synthetase, } \\
\text { cytoplasmic }\end{array}$ & gi|57524852 & AARS & $102.00 / 5.68$ & 11 & $14 / 189$ & 272 & -1.47 & $4.5 E-03$ \\
\hline 22 & Homogentisate 1,2-dioxygenase & gi|50729534 & HGD & $50.12 / 6.35$ & 34 & $17 / 279$ & 397 & 1.79 & $3.6 \mathrm{E}-03$ \\
\hline 24 & $\begin{array}{l}\text { Betaine-homocysteine } \\
\text { S-methyltransferase } 1\end{array}$ & gi|50755288 & BHMT & $45.55 / 7.56$ & 52 & $27 / 265$ & 507 & 1.78 & $3.8 \mathrm{E}-04$ \\
\hline 29 & Protein disulfide-isomerase A4 & gi|57530768 & PDIA4 & $71.29 / 4.96$ & 11 & $7 / 228$ & 115 & -1.62 & $2.8 \mathrm{E}-03$ \\
\hline 32 & $\begin{array}{l}\text { Aspartate aminotransferase, } \\
\text { cytoplasmic }\end{array}$ & gi|809192 & GOT1 & $46.00 / 8.26$ & 54 & $32 / 245$ & 943 & 1.44 & $4.4 \mathrm{E}-02$ \\
\hline 33 & Cystathionase & gi|118094764 & $\mathrm{CTH}$ & $44.56 / 6.86$ & 38 & $17 / 233$ & 335 & 1.47 & $4.8 \mathrm{E}-02$ \\
\hline 41 & $\begin{array}{l}\text { Betaine-homocysteine } \\
\text { S-methyltransferase }\end{array}$ & gi|50755288 & BHMT & $45.55 / 7.56$ & 32 & $18 / 259$ & 316 & 1.49 & $1.4 \mathrm{E}-02$ \\
\hline 42 & Prolyl-4-hydroxylase & gi|63739 & $\mathrm{P} 4 \mathrm{HB}$ & $55.17 / 4.66$ & 13 & $7 / 250$ & 146 & -1.89 & $1.1 \mathrm{E}-03$ \\
\hline 43 & $\begin{array}{l}\text { Protein disulfide-isomerase } \\
\text { A3 precursor }\end{array}$ & gi|45383890 & PDIA3 & $56.55 / 5.76$ & 28 & $24 / 238$ & 589 & -1.42 & $4.4 \mathrm{E}-03$ \\
\hline 45 & Elongation factor 2 & gi|45382453 & EEF2 & $96.34 / 6.40$ & 6 & $7 / 255$ & 94 & -1.40 & $2.1 E-03$ \\
\hline \multicolumn{10}{|c|}{ Lipid and vitamin metabolism } \\
\hline 28 & Retinal dehydrogenase 1 & gi|45383031 & ALDH1A1 & $56.40 / 7.49$ & 16 & $14 / 229$ & 111 & 1.41 & $3.7 E-02$ \\
\hline 35 & $\begin{array}{l}\text { 3-oxo-5- } \beta \text {-steroid } \\
\text { 4-dehydrogenase isoform } 2\end{array}$ & gi|118082901 & AKR1D1 & $50.02 / 9.32$ & 52 & $26 / 256$ & 655 & 1.44 & $4.4 \mathrm{E}-02$ \\
\hline 38 & Regucalcin & gi|45382019 & RGN & $33.67 / 5.77$ & 75 & $39 / 286$ & 1279 & 1.41 & $2.7 E-03$ \\
\hline 48 & Apolipoprotein A-I & gi|211159 & APOA1 & $30.67 / 5.58$ & 10 & $3 / 250$ & 59 & -1.42 & $3.6 \mathrm{E}-02$ \\
\hline 57 & Fatty acid-binding protein, liver & gi|45383728 & FABP1 & $14.30 / 7.74$ & 66 & $17 / 286$ & 461 & -1.90 & $2.6 E-03$ \\
\hline \multicolumn{10}{|c|}{ Nucleotide metabolism } \\
\hline 8 & $\begin{array}{l}\text { Bifunctional purine biosynthesis } \\
\text { protein PURH }\end{array}$ & gi|28373618 & ATIC & $67.05 / 8.54$ & 31 & $30 / 230$ & 364 & 2.46 & $1.7 \mathrm{E}-03$ \\
\hline 53 & Dihydropyrimidinase & gi|1 18087274 & DPYS & $69.50 / 6.42$ & 4 & $2 / 265$ & 70 & 1.51 & $4.5 E-02$ \\
\hline 54 & Nucleoside diphosphate kinase & gi|2827446 & NME4 & $17.54 / 7.11$ & 65 & $27 / 277$ & 492 & 1.43 & $6.8 \mathrm{E}-03$ \\
\hline
\end{tabular}

${ }^{a}$ Spot no. corresponds to the number of protein spots in Fig. 1. Protein name is given when proteins were identified by LC-Chip ESI-QTOF MS. Accession no. is the unique number given to mark the entry of a protein in the database NCBInr. Theoretical molecular weight $(\mathrm{Mr})$ and isoelectric point ( $\mathrm{pl}$ ) of the identified proteins are retrieved from the protein database of NCBInr. Sequence coverage is the ratio of the number of amino acids in every peptide that matches with the mass spectrum divided by the total number of amino acids in the protein sequence. Matched peptide is the number of paring an experimental fragmentation spectrum to a theoretical segment of protein and searched peptide is the total searched peptide. Peptides were identified from the liver of AA broiler chickens based on Mascot scores (Additional file 3: Table S3) . Mascot scores are derived from ion scores as a non-probabilistic basis for ranking protein hits. Av. ratio and $p$ value are calculated using DeCyder software version 7.0 


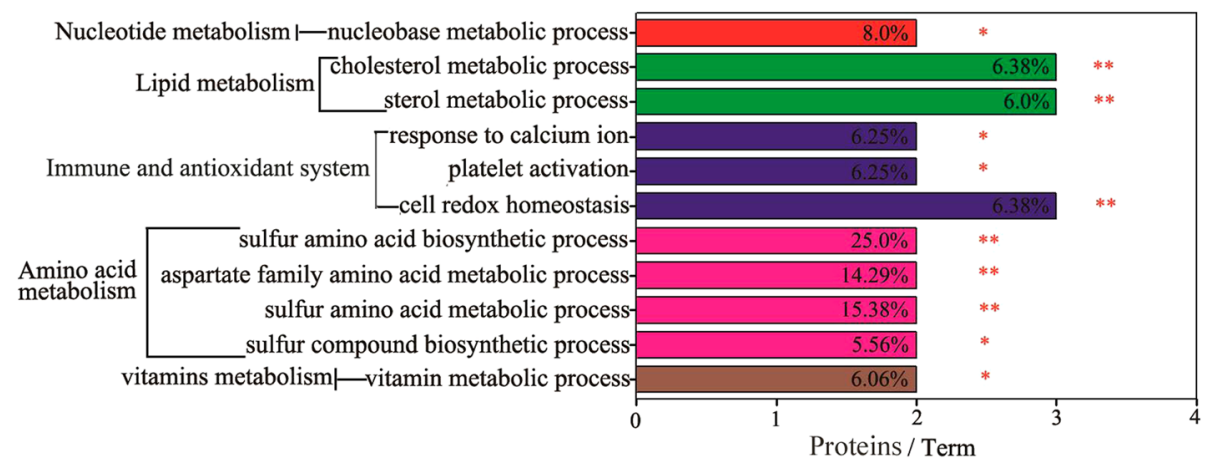

Fig. 2 Functional enrichment analysis of the proteins of differential abundance in the livers of broilers fed the probiotic E. faecium. using ClueGO software. ${ }^{*}$ and ${ }^{* *}$ mean $p<0.05$ and $p<0.01$ levels of significance

related to glycolysis/gluconeogenesis, retinol metabolism, and amino acid metabolism. Of them, the metabolism of glycine, serine, threonine, cysteine, methionine and tyrosine and the retinol metabolism pathway were enhanced according to the up-regulated proteins that were enriched (Tables 1 and 2).

\section{Protein and protein interaction analysis}

Of the 58 differentially expressed protein spots, twentysix proteins were recognized as nodes with various relationships in the biological interaction networks (BIN) using the online tools of STRING 10.0 (Fig. 3). The node proteins were separated into six clusters, connected by twelve key node proteins (ENO1, EEF2, HSP90B1, PDIA3, PDIA4, P4HB, CAT, ALB, APOA1, ATIC, GOT1 and BHMT). The up-regulation of $\mathrm{CTH}, \mathrm{GOT} 1$ and BHMT may be related to cysteine and methionine metabolism, while the down-regulation of HSP90B1 together with PDIA3, PDIA4, P4HB and EEF2 involves protein processing in the endoplasmic reticulum. The down-regulation of APOA1 and FABP1 may influence the uptake, transportation and deposition of fatty acids. The interaction of up-regulation of ALB and downregulation of FGB, FGG and LTF probably plays a vital role in immunity and antioxidation. Other BIN proteins (PGK1, ENO1, PCK2 and ATIC) may function in carbohydrate metabolism. Of these, HSP90B1, ENO1, EEF2, and ALB are the most important hub proteins in the BIN system.

\section{Validation of proteins of differential abundance by qPCR} Of the liver proteins with differential abundance, ten proteins that played an important role in nutrient metabolism (amino acid and lipid metabolism) and the immune system were selected to validate their expression at the level of mRNA (Fig. 4). The results showed that six of ten, CAT (spots 12 and 13), BHMT (spots 24 and 41), PDIA4 (spot 29), GOT1 (spot 32), CTH (spot 33), and PDIA3 (spot 43), were consistent with their mRNA expression levels. The similar expression pattern at the transcript level indicates a prospective opportunity for reverse genetic research through gene manipulation at different developmental stages of chickens. The other four genes, FGB (spot 14), FGG (spots 16 and 18), P4HB (spot 42) and APOA1 (spot 48), showed an inconsistent pattern between the mRNA and protein expression level.

\section{Discussion}

The liver is the major metabolic organ in the body and it modulates the complexity of the processes of metabolism. Understanding the alterations that occur in hepatic metabolism following the administration of a probiotic to poultry, is an important aspect of delineating the mechanism of probiotic action and determining use

Table 2 Enriched KEGG pathway-based sets of proteins of differential abundance in the liver of AA broiler chickens fed the probiotic

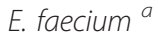

\begin{tabular}{lllll}
\hline Pathway name & Count & Protein & $p$ value & $q$ value \\
\hline Glycolysis/Gluconeogenesis & 3 & ADH6, PGK1, ENO1 & $1.67 \mathrm{E}-03$ & $8.33 \mathrm{E}-03$ \\
Glycine, serine and threonine metabolism & 2 & BHMT, CTH & $1.29 \mathrm{E}-02$ & $6.46 \mathrm{E}-02$ \\
Cysteine and methionine metabolism & 3 & BHMT, CTH, GOT1 & $3.58 \mathrm{E}-04$ & $1.79 \mathrm{E}-03$ \\
Tyrosine metabolism & 3 & ADH6, GOT1, HGD & $5.35 \mathrm{E}-04$ & $2.68 \mathrm{E}-03$ \\
Retinol metabolism & 2 & ADH6, ALDH1A1 & $9.49 \mathrm{E}-03$ & $4.74 \mathrm{E}-02$
\end{tabular}

${ }^{a}$ The number of count refers to the amount of proteins which are part of the extended network and appear as part of the pathway. $p$ values are calculated according to a hypergeometric test, $q$ values represent $p$ values corrected for multiple testing using the false discovery rate method. Protein refers to the proteins involved in the corresponding pathway 


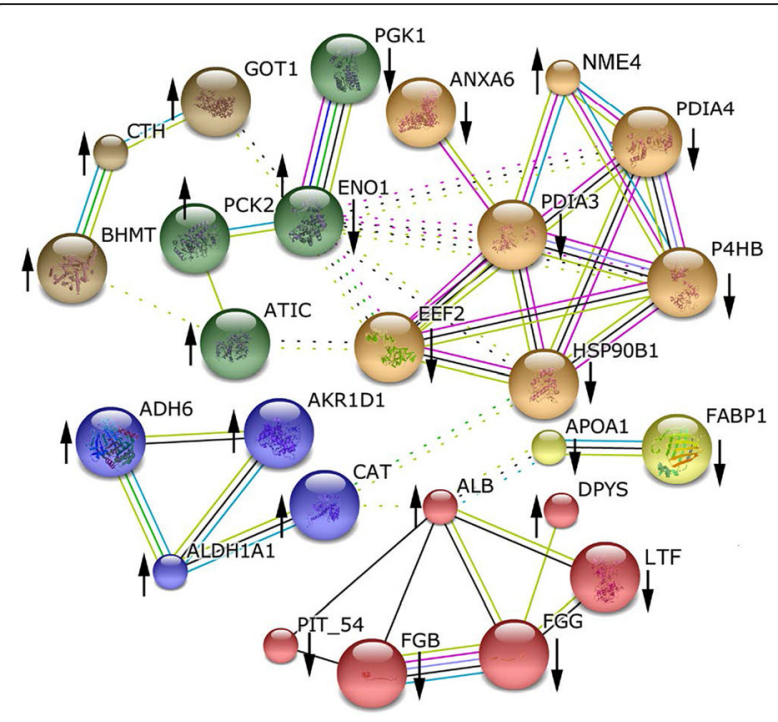

Fig. 3 Biological interaction network of the proteins of differential abundance in the livers of broilers fed the probiotic E. faecium. Red lines indicate fusion evidence, green lines indicate neighborhood evidence, blue lines indicate co-occurrence evidence, purple lines indicate experimental evidence, yellow lines indicate text mining evidence light blue lines indicate database evidence, and black lines indicate coexpression evidence. $\uparrow$ and $\downarrow$ indicate up-regulated and downregulated proteins in the livers of broilers fed the probiotic $E$. faecium, respectively

strategies. The birds sampled in this study, used their feed more efficiently [9] and had improved carcass yield and quality [14].

The broiler chickens fed E. faecium had a significantly different hepatic protein expression profile than birds not exposed to the probiotic. Dietary supplementation with E. faecium significantly changed the immune and antioxidant system and the metabolism of carbohydrates, amino acids, nucleotides, vitamins, and lipids in the liver. In this regard, sulphur amino acids, vitamin and cellular hormone metabolism, sulphur compound biosynthesis and protein tetramerization were enhanced following feeding with E. faecium. In contrast, proteins involved in calcium ion flux, cell redox homeostasis and platelet activation were down-regulated. These results indicate that dietary supplementation with E. faecium may alter the partitioning of nutrients, thus facilitating optimal nutrient utilization.

The liver plays an important immunological role [27]. When inflammation occurs, the acute phase response often appears in the liver [28]. This response is a natural systemic defense to help protect the body against infections, neoplasm, stress, immune disorders, trauma or parasites [29]. Acute phase proteins (APPs), synthesized in the liver during the acute phase response [30], can be divided as positive or negative based on increase or decline of their concentrations during inflammatory responses
[31, 32]. Positive APPs in avian species include ceruloplasmin, $\alpha 1$-acid glycoprotein, amyloid A, transferrin (LTF), mannan-binding protein, haptoglobin, hemopexin, fibrinogen and fibronectin [33], while albumin (ALB) was reported to a negative APP in poultry [34]. In this study, down-regulation of positive APPs including LTF, FGB and FGG and up-regulation of negative APP, such as ALB, suggest a possible relationship between supplementation with E. faecium and decreased inflammation. This result is consistent with that observed in the intestinal mucosa and immune organ indexes of these broilers [9]. Moreover, HSP90B1 is an essential immune chaperone, regulating innate and adaptive immunity [35]. Therefore, downregulation of HSP90B1 (spots 40, 46, and 51) and differential expression of APPs in the liver of treated broilers suggests that supplementation with E. faecium may improve broiler health as the concentration of APPs may be useful for monitoring domestic fowl health [36]. Under normal physiological conditions, reduced inflammation will result in more efficience in animal production [37]. Chickens with high concentrations of positive APPs had growth depression and decreased meat quality because of repartitioning of nutrients away from muscle protein deposition and growth to APPs synthesis, in response to inflammation $[38,39]$. In our study, the higher efficiency of production in the broilers fed with E. faecium may be due to the redistribution of nutrients away from the immune response resulting in increased availability of nutrients for growth and development.

Oxidative stress can induce cellular damage and cause disruption of normal cell signaling by over reactive oxygen species [40]. Antioxidant enzymes can protect cells from oxidative stress damage [41, 42]. High-level expression of antioxidant enzymes, i.e. CAT (spots 12 and 13), epoxide hydrolase 2 (EPHX2, spot 34), and glutathione S-transferase 2 (GSTM2, spot 50), suggests that the broilers fed E. faecium have synthesized more antioxidants to protect their livers from oxidative damage.

Dietary carbohydrates provide more than half the energy requirement, demonstrating the importance of hepatic carbohydrate metabolism to broiler chicken health and production. Proteins related to carbohydrate metabolism that were up-regulated in the liver of chickens fed E. faecium, included ENO1 (spots 20, 21, 23, 25, 26, and 36), phosphoglycolate phosphatase (PGP, spot 37), ADH6 (spot 55), and phosphoenolpyruvate carboxykinase (PCK2, spot 56). These proteins are involved in gluconeogenesis and glycolysis. However, phosphoglycerate kinase which is also involved in glycolysis (PGK1, spot 56) was down-regulated. The results suggest that supplementation of E. faecium significantly changed the carbohydrate metabolic pathways. ENO1 is a multifunctional protein that plays roles in glycolysis and carbohydrate degradation. Human ENO1 has 32 post-translational 

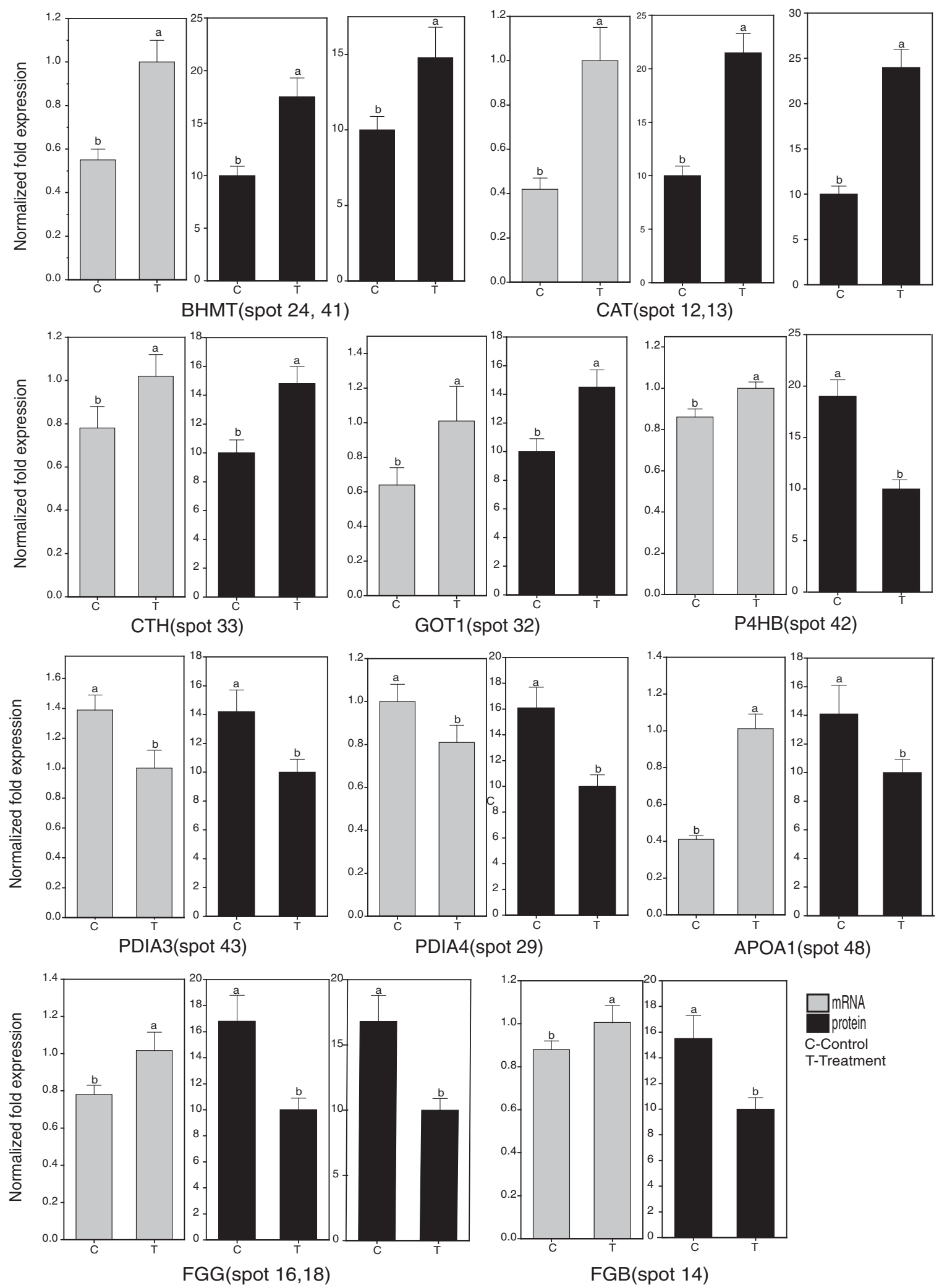

Fig. 4 Validation using qPCR of ten proteins of differential abundance at the mRNA level in the livers of broilers fed the probiotic E. faecium. Samples were normalized with the reference genes $28 \mathrm{~S}$ rRNA and actin. Gray and black histograms show the levels of mRNA and protein, respectively 
modification sites for phosphorylation, acetylation and ubiquitination (http://www.uniprot.org/uniprot/P06733\# publications), and its functional roles depend on subcellular localization, post-translational modifications and the concentrations of modified protein [43]. In this study, eight differential protein spots of ENO1 were identified in the liver of broilers as reported in Plasmodium yoelii [44], including six up-regulated and two down-regulated. It suggests that supplementation of $E$. faecium may change the abundance and diversity of modified ENO1, and its physiological functions as a consequence. However, how and which ENO1 isoforms play the physiological roles requires further study.

Proteins, accounting for about 18 percent of the body weight of animals, are vital constituents of all cells and play important roles in many biological activities. In this study, the proteins related to amino acid and protein metabolism were identified to differentially express, such as alanyltRNA synthetase (AARS, spot 17); PDIA4 (spot 29), P4HB (spot 42), PDIA3 (spot 43) and EEF2 (spot 45). These proteins are principally involved in the synthesis of proteins [45], rearrangement of intrachain and interchain disulphide bonds [46], and polypeptide chain elongation [47].

Lipids play physiologically important roles in regulatory metabolism, metabolic energy production and as constituents of cell membranes [48]. APOA1 (spot 48) constitutes the high density lipoprotein complex with lipids in the plasma, and transports lipids from tissue to liver for excretion. FABP1 (spot 57) primarily binds long-chain fatty acids and hydrophobic ligands [49] and is associated with fatty acid uptake [50]. The downregulation of APOA1 and FABP1 in the liver may be related to a decline in abdominal fat percentage which was observed in these birds [14]. Vitamins play a role as metabolic catalysts in the form of coenzymes. The differential proteins involved in metabolism of vitamins and nucleotides were identified in the liver of broilers fed $E$. faecium. RGN plays major roles in L-ascorbic acid biosynthesis and regulates hepatic cell functions [51]. Upregulated RGN in the liver of broilers fed E. faecium may be linked to enhanced vitamin $C$ synthesis and increased broiler immunity.

Proteins serve as fundamental elements in the living cell but do not exist independently [52]. KEGG pathway enrichment analysis and BIN analysis of differentially expressed proteins is the best way to perform functional analysis [53, 54]. Significantly enriched biological pathways of glycolysis/gluconeogenesis, retinol metabolism, and amino acid metabolism were observed in this study, indicating their central roles in hepatic metabolic enhancement. The proteins included in the BIN were mainly involved in the immune and antioxidant system and metabolism of carbohydrates, amino acids, proteins, nucleotides, lipids and vitamins. The complex network would appear to indicate that supplementation of broilers with $E$. faecium is related to the improved cysteine and methionine metabolism, reduced lipid deposition, immunoinflammatory responsiveness, and enhanced protein processing in hepatic endoplasmic reticulum. Thus, the important hub proteins in the interaction network, HSP90B1, FGG, FGB and APOA1 play the vital role in improving immune function and metabolism of the liver in broilers fed with E. faecium. Some of the key node proteins that were highly linked in the BIN were validated at a gene level. Proteins BHMT, CAT, CTH, GOT1, PDIA3 and PDIA4 with abundances corresponding to mRNA levels may represent potential targets for genetic manipulation. However, possible reasons for these inconsistent results might include: 1) delayed outcomes of some biological processes, such as transcription or translation initiation, elongation efficiency, mRNA stability, or splicing [55-57]; 2) post-translational modifications, e.g. phosphorylation, or proteolysis of core components of the translation machinery [58-60]; 3) time delays between responses at the mRNA and protein levels; and 4) different degradation rates of proteins and mRNAs [61, 62]. Manipulation of these genes may require further experimental information.

\section{Conclusion}

This study assists in the unraveling of the molecular effects of dietary probiotics in the livers of broiler chickens by using proteomics technology. Fifty differentially expressed proteins were identified in the treated broilers, and most of them are related to the metabolism of glycine, serine, threonine, cysteine, methionine and tyrosine and inflammatory responses. These results suggest that dietary supplementation of E. faecium may alter the partitioning of nutrients in the body and facilitate optimal utilization of nutrients by broilers. This study extends our previous knowledge of the mechanism of dietary probiotic action and provides new findings for research and future probiotic development.

\section{Additional files}

Additional file 1: Table S1. The composition of broiler chickens starter and grower diets (g/ $/ \mathbf{k g})$. (DOC $43 \mathrm{~kb}$ )

Additional file 2: Table S2. The primer sequences used for qPCR analysis of the differentially expressed proteins of the liver of $A A$ broiler chickens. (DOC $37 \mathrm{~kb}$ )

Additional file 3: Table S3. Peptides identified from the liver of AA broiler chickens based on Mascot scores. (DOC $103 \mathrm{~kb}$ )

\section{Abbreviations}

2-D DIGE: two-dimensional fluorescence difference gel electrophoresis; AARS: Alanyl-tRNA synthetase; ADH6: Alcohol dehydrogenase 6; AKR1D1: 3-oxo-5- $\beta$-steroid 4-dehydrogenase isoform 2; ALB: serum albumin precursor; ALDH1A1: retinal dehydrogenase 1; APOA1: apolipoprotein A-l; APPs: acute phase proteins; ATIC: bifunctional purine biosynthesis protein $\mathrm{PURH}$; BHMT: betaine-homocysteine S-methyltransferase 1; BIN: biological 
interaction networks; CAAS: Chinese Academy of Agricultural Sciences; CAT: catalase; CTH: cystathionase; DPYS: dihydropyrimidinase; E. faecium: enterococcus faecium; EEF2: elongation factor 2; ENO1: a-Enolase; EPHX2: epoxide hydrolase 2; FABP1: Fatty acid-binding protein liver; FGB: fibrinogen $\beta$ chain; FGG: fibrinogen gamma chain; GO: Gene Ontology; GOT1: aspartate aminotransferase cytoplasmic; GSTM2: glutathione S-transferase 2; HGD: homogentisate 1,2-dioxygenase; HSP90B1: HSP108; KEGG: Kyoto encyclopedia of genes and genomes database; LTF: transferrin; MS: mass spectrometry; P4HB: prolyl-4-hydroxylase; PCK2: phosphoenolpyruvate carboxykinase; PDIA3: protein disulfide-isomerase A3 precursor; PDIA4: protein disulfide-isomerase A4; PGK1: phosphoglycerate kinase; PGP: phosphoglycolate phosphatase; PIT 54: PIT 54 protein; RGN: regucalcin; STRING: Search Tool for the Retrieval of Interacting Genes.

\section{Competing interests}

The authors declared that they had no competing interests.

\section{Authors' contribution}

BY and GHL designed the experiment, coordinated and supervised the research. AJZ, JJL and KM made the greatest contribution to the conduction of the experiment and analysis of data. SZ and LXNW assisted in the collection of samples. AJZ drafted the manuscript with the assistance of JKL, WHC and WLB. All authors read and approved the final manuscript.

\section{Acknowledgments}

This work was supported by the National High-Tech Research and Development Program (863 Program, 2013AA102803) and the National Chicken Industry Technology System (CARS-42) and the National Natural Science Foundation (31101731) of China. The authors are grateful to GE Healthcare and Bio-Rad for their excellent technical assistance.

\section{Author details}

'Key Laboratory of Feed Biotechnology of Ministry of Agriculture, Feed Research Institute, Chinese Academy of Agricultural Sciences, Beijing 100081, People's Republic of China. ${ }^{2}$ Key Laboratory of Pollinating Insect Biology of Ministry of Agriculture, Institute of Apicultural Research, Chinese Academy of Agricultural Sciences, Beijing 100093, People's Republic of China. ${ }^{3}$ School of Agriculture and Food Sciences, University of Queensland, Gatton, QLD 4343, Australia.

Received: 14 July 2015 Accepted: 6 January 2016

Published online: 01 February 2016

\section{References}

1. Ferreira CL, Salminen S, Grzeskowiak L, Brizuela M, Sanchez L, Carneiro H, et al. Terminology concepts of probiotic and prebiotic and their role in human and animal health. Rev Salud Anim. 2011;33(3):137-46.

2. Charalampopolus DRAR, editor. Prebiotics and probiotics science and technology. New York: Springer; 2009.

3. Pirgozliev V, Bravo D, Rose SP. Rearing conditions influence nutrient availability of plant extracts supplemented diets when fed to broiler chickens. J Anim Physiol Anim Nutr (Berl). 2014;98(4):667-71.

4. Czerwinski J, Hojberg O, Smulikowska S, Engberg RM, Mieczkowska A. Influence of dietary peas and organic acids and probiotic supplementation on performance and caecal microbial ecology of broiler chickens. Br Poult Sci. 2010;51(2):258-69.

5. Phillips I, Casewell M, Cox T, De Groot B, Friis C, Jones R, et al. Does the use of antibiotics in food animals pose a risk to human health? A critical review of published data. J Antimicrob Chemother. 2004;53(1):28-52.

6. Rahkila R, Johansson P, Sade E, Bjorkroth J. Identification of enterococci from broiler products and a broiler processing plant and description of Enterococcus viikkiensis sp. nov. Appl Environ Microbiol. 2011;77(4):1 196-203.

7. Capcarova M, Weiss J, Hrncar C, Kolesarova A, Pal G. Effect of Lactobacillus fermentum and Enterococcus faecium strains on internal milieu, antioxidant status and body weight of broiler chickens. J Anim Physiol Anim Nutr (Berl). 2010;94(5):e215-24.

8. Zhao X, Guo Y, Guo S, Tan J. Effects of Clostridium butyricum and Enterococcus faecium on growth performance, lipid metabolism, and cecal microbiota of broiler chickens. Appl Microbiol Biotechnol. 2013;97(14):6477-88.
9. Luo J, Zheng A, Meng K, Chang W, Bai Y, Li K, et al. Proteome changes in the intestinal mucosa of broiler (Gallus gallus) activated by probiotic Enterococcus faecium. J Proteomics. 2013;91:226-41.

10. Samli HE, Senkoylu N, Koc F, Kanter M, Agma A. Effects of Enterococcus faecium and dried whey on broiler performance, gut histomorphology and intestinal microbiota. Arch Anim Nutr. 2007:61(1):42-9.

11. Carina Audisio M, Oliver G, Apella MC. Protective effect of Enterococcus faecium J96, a potential probiotic strain, on chicks infected with Salmonella Pullorum. J Food Prot. 2000;63(10):1333-7.

12. Levkut M, Revajova V, Laukova A, Sevcikova Z, Spisakova V, Faixova Z, et al. Leukocytic responses and intestinal mucin dynamics of broilers protected with Enterococcus faecium EF55 and challenged with Salmonella Enteritidis. Res Vet Sci. 2012;93(1):195-201.

13. Samli HE, Dezcan S, Koc F, Ozduven ML, Okur AA, Senkoylu N. Effects of Enterococcus faecium supplementation and floor type on performance, morphology of erythrocytes and intestinal microbiota in broiler chickens. Br Poult Sci. 2010;51(4):564-8

14. Zheng A, Luo J, Meng K, Li J, Zhang S, Li K, et al. Proteome changes underpin improved meat quality and yield of chickens (Gallus gallus) fed the probiotic Enterococcus faecium. BMC Genomics. 2014;15:1167.

15. Grune T, Reinheckel T, Joshi M, Davies KJ. Proteolysis in cultured liver epithelial cells during oxidative stress. Role of the multicatalytic proteinase complex, proteasome. J Biol Chem. 1995;270(5):2344-51.

16. Rosebrough RW, McMurtry JP, Mitchell AD, Steele NC. Chicken hepatic metabolism in vitro. Protein and energy relations in the broiler chicken-VI. Effect of dietary protein and energy restrictions on in vitro carbohydrate and lipid metabolism and metabolic hormone profiles. Comp Biochem Physiol B. 1988;90(2):311-6.

17. Wang $T, L i A, Y i$ J, Tao H, Wang Y. Screening, identification and characterization of Enterococcus faecium for feed use. J Chin Cereal Oil Assoc. 2010;25:89-97.

18. Wang T, Li A, Tao H, Yi J. Study on the production technology and properties of microencapsulated Enterococcus faecium. J Northwest A\&F Univ (Nat Sci Ed). 2009;37:51-62.

19. Zhang L, Yue HY, Zhang HJ, Xu L, Wu SG, Yan HJ, et al. Transport stress in broilers: I. Blood metabolism, glycolytic potential, and meat quality. Poult Sci. 2009;88(10):2033-41.

20. Zhang L, Yue HY, Wu SG, Xu L, Zhang HJ, Yan HJ, et al. Transport stress in broilers. II. Superoxide production, adenosine phosphate concentrations, and mRNA levels of avian uncoupling protein, avian adenine nucleotide translocator, and avian peroxisome proliferator-activated receptor-gamma coactivator-1alpha in skeletal muscles. Poult Sci. 2010;89(3):393-400.

21. Lu Y, Liu X, Shi S, Su H, Bai X, Cai G, et al. Bioinformatics analysis of proteomic profiles during the process of anti-Thy 1 nephritis. Mol Cell Proteomics. 2012;11(4):M111 008755.

22. Begna $\mathrm{D}$, Fang $\mathrm{Y}$, Feng $\mathrm{M}$, Li J. Mitochondrial proteins differential expression during honeybee (Apis mellifera L.) queen and worker larvae caste determination. J Proteome Res. 2011;10(9):4263-80.

23. Bindea G, Mlecnik B, Hackl H, Charoentong P, Tosolini M, Kirilovsky A, et al. ClueGO: a Cytoscape plug-in to decipher functionally grouped gene ontology and pathway annotation networks. Bioinformatics. 2009;25(8): $1091-3$.

24. Szklarczyk D, Franceschini A, Kuhn M, Simonovic M, Roth A, Minguez P, et al. The STRING database in 2011: functional interaction networks of proteins, globally integrated and scored. Nucleic Acids Res. 2011; 39(Database issue):D561-8.

25. Livak KJ, Schmittgen TD. Analysis of relative gene expression data using realTime quantitative PCR and the $2^{-\Delta \Delta C T}$ method. Methods. 2001;25(4):402-8.

26. Bustin SA, Benes V, Garson JA, Hellemans J, Huggett J, Kubista M, et al. The MIQE guidelines: minimum information for publication of quantitative realtime PCR experiments. Clin Chem. 2009;55(4):611-22.

27. Nakamura K, Ibaraki Y, Mitarai Z, Shibahara T. Comparative pathology of heart and liver lesions of broiler chickens that died of ascites, heart failure, and others. AVian Disease. 1999;43(3):526-32.

28. Saini PK, Webert DW. Application of acute phase reactants during antemortem and postmortem meat inspection. J Am Vet Med Assoc. 1991; 198(11):1898-901

29. Georgieva TM. Similarities and differences between haptoglobin in mammals and PIT54 in poultry-a review. Bulg J Vet Med. 2010; 13(1):1-12.

30. Kaneko J, editor. Serum proteins and the dysproteinemias. San Diego: Academic; 1977. 
31. Koj A. Definition and classification of acute-phase proteins. Amsterdam: Elsevier; 1985.

32. Gruys E, Obwolo MJ, Toussaint MJM. Diagnostic significance of the major acute phase proteins in veterinary clinical chemistry: a review. Veterinary Bulletin. 1994;64:1009-18.

33. Chamanza R, Toussaint MJ, van Ederen AM, van Veen L, Hulskamp-Koch C, Fabri TH. Serum amyloid A and transferrin in chicken. A preliminary investigation of using acute-phase variables to assess diseases in chickens. Vet Q. 1999;21(4):158-62.

34. Kaneko JJ. Chapter 5 - Serum Proteins and the Dysproteinemias. In: Bruss JJKWHL, editor. Clinical Biochemistry of Domestic Animals (5). San Diego: Academic; 1997. p. 117-38.

35. Schild H, Rammensee H. gp96-the immune system's Swiss army knife. Nat Immunol. 2000;1(2):100-1.

36. Chamanza R, Vanveen L, Tivapasi MT, Toussaint MJ. Acute phase proteins in the domestic fowls. Worlds Poult. Sci. J. 1999; 55(1):61-71.

37. Williams NH, Stahly TS, Zimmerman DR. Effect of chronic immune system activation on the rate, efficiency, and composition of growth and lysine needs of pigs fed from 6 to 27 kg. J Anim Sci. 1997;75(9):2463-71.

38. Klasing KC, Laurin DE, Peng RK, Fry DM. Immunologically mediated growth depression in chicks: influence of feed intake, corticosterone and interleukin-1. J Nutr. 1987;117(9):1629-37.

39. Klasing KC, Johnstone BJ. Monokines in growth and development. Poult Sci. 1991;70(8):1781-9.

40. Blokhina O, Virolainen E, Fagerstedt KV. Antioxidants, oxidative damage and oxygen deprivation stress: a review. Ann Bot. 2003;91:179-194.

41. Halliwell B, Gutteridge JM. Oxygen free radicals and iron in relation to biology and medicine: some problems and concepts. Arch Biochem Biophys. 1986;246(2):501-14.

42. Altan O, Pabuccuoglu A, Altan A, Konyalioglu S, Bayraktar H. Effect of heat stress on oxidative stress, lipid peroxidation and some stress parameters in broilers. Br Poult Sci. 2003;44(4):545-50.

43. Merkulova T, Dehaupas M, Nevers MC, Creminon C, Alameddine $H$, Keller A. Differential modulation of alpha, beta and gamma enolase isoforms in regenerating mouse skeletal muscle. Eur J Biochem. 2000; 267(12):3735-43.

44. Pal-Bhowmick I, Vora HK, Jarori GK. Sub-cellular localization and posttranslational modifications of the Plasmodium yoelii enolase suggest moonlighting functions. Malar J. 2007;6:45.

45. Berg JM, Tymoczko JL, Stryer L, editors. Biochemistry. 5th ed. New York: W. H. Freeman and Company; 2002.

46. Galligan JJ, Petersen DR. The human protein disulfide isomerase gene family. Hum Genomics. 2012;6:6.

47. Redpath NT, Foulstone EJ, Proud CG. Regulation of translation elongation factor-2 by insulin via a rapamycin-sensitive signalling pathway. EMBO J. 1996;15(9):2291-7.

48. Hulbert AJ, Turner N, Storlien LH, Else PL. Dietary fats and membrane function: implications for metabolism and disease. Biol Rev Camb Philos Soc. 2005;80(1):155-69.

49. Keler T, Khan S, Sorof S. Liver Fatty Acid Binding Protein and Mitogenesis in Transfected Hepatoma Cells. In: Honn K, Nigam S, Marnett L, editors. Eicosanoids and Other Bioactive Lipids in Cancer, Inflammation, and Radiation Injury 2. US: Springer; 1997. p. 517-24. vol. 400.

50. Vogel Hertzel A, Bernlohr DA. The Mammalian Fatty Acid-binding Protein Multigene Family: Molecular and Genetic Insights into Function. Trends in Endocrinology \& Metabolism. 2000;11(5):175-80.

51. Yamaguchi M. Role of regucalcin in maintaining cell homeostasis and function (Review). International Journal of Molecular Medicine. 2005;15(3): 371-89.

52. Vidal M. A unifying view of 21 st century systems biology. FEBS Lett. 2009; 583(24):3891-4.

53. Ashburner M, Ball CA, Blake JA, Botstein D, Butler H, Cherry JM, et al. Gene ontology: tool for the unification of biology. The Gene Ontology Consortium. Nat Genet. 2000;25(1):25-9.

54. Vidal M, Cusick ME, Barabasi AL. Interactome networks and human disease. Cell. 2011;144(6):986-98.

55. Breaker RR. Ancient, giant riboswitches at atomic resolution. Nat Struct Mol Biol. 2012;19(12):1208-9.

56. Smith AM, Fuchs RT, Grundy FJ, Henkin TM. Riboswitch RNAs: regulation of gene expression by direct monitoring of a physiological signal. RNA Biol. 2010;7(1):104-10.
57. Mandal M, Breaker RR. Gene regulation by riboswitches. Nat Rev Mol Cell Biol. 2004;5(6):451-63.

58. Bartel DP, Chen CZ. Micromanagers of gene expression: the potentially widespread influence of metazoan microRNAs. Nat Rev Genet. 2004;5(5): 396-400.

59. Abaza I, Gebauer F. Trading translation with RNA-binding proteins. RNA. 2008;14(3):404-9.

60. Gebauer F, Hentze MW. Molecular mechanisms of translational control. Nat Rev Mol Cell Biol. 2004;5(10):827-35.

61. de Sousa AR, Penalva LO, Marcotte EM, Vogel C. Global signatures of protein and mRNA expression levels. Mol Biosyst. 2009;5(12):1512-26.

62. Valencia-Sanchez MA, Liu J, Hannon GJ, Parker R. Control of translation and mRNA degradation by miRNAs and siRNAs. Genes Dev. 2006;20(5):515-24.

\section{Submit your next manuscript to BioMed Central and we will help you at every step:}

- We accept pre-submission inquiries

- Our selector tool helps you to find the most relevant journal

- We provide round the clock customer support

- Convenient online submission

- Thorough peer review

- Inclusion in PubMed and all major indexing services

- Maximum visibility for your research

Submit your manuscript at www.biomedcentral.com/submit
Biomed Central 